\title{
Second-Order Perturbation Analysis of Low-Amplitude Traveling Waves in a Periodic Chain With Quadratic and Cubic Nonlinearity
}

\author{
Smruti R. Panigrahi*a, Brian F. Feeny ${ }^{a}$, and Alejandro R. Diaz ${ }^{a}$ \\ ${ }^{a} 428$ S. Shaw Lane, Michigan State University, East Lansing, MI, 48824, USA
}

\begin{abstract}
Traveling waves in one-dimensional nonlinear periodic structures are investigated for low-amplitude oscillations using perturbation analysis. We use second-order multiple scales analysis to capture the effects of the quadratic nonlinearity. Comparisons with the linear and cubical nonlinear cases are presented in the propagation and attenuation of the wave as well as the dispersion relationships, group and phase velocities and their dependence on wave number and amplitude of oscillation. Quadratic nonlinearity is shown to have a significant effect on the wave propagation behavior in the chain. Given the wavenumber, the quadratic nonlinearity (with nonzero linear and zero cubic terms) has shown to produce higher frequencies than linear system. At lower wave numbers, the quadratic system has higher phase and group velocities than the linear case. At higher wave numbers, however, the group velocities for the quadratic system are lower as compared to the linear case.
\end{abstract}

\section{Research highlights}

$\rightarrow$ A second-order perturbation analysis of single-wave propagation in a periodic nonlinear chain is presented using both discrete and continuum analysis.

$\rightarrow$ While the first-order multiple scales captures the cubic effect alone, the second-order analysis uncovers the quadratic effect.

$\rightarrow$ The quadratic nonlinearity shows to affect the wave propagation velocities and phases.

Keywords: Traveling waves, second-order perturbation, method of multiple scales, amplitude-dependent dispersion, nonlinear waveguides, propagation, attenuation, metamaterials.

\section{INTRODUCTION}

Metamaterials have been of growing interest for achieving certain desirable properties such as band gaps, negative refractive indices, phonon tunneling, and phonon focusing [1]. Engineering applications of these properties are in waveguides, acoustic filters, acoustic mirrors, transducers, etc. [2]-[8]. Various types of periodic media have been studied, including one-dimensional undamped mass-spring chains [9]-[12], strongly nonlinear contact in beaded systems [13], kink dynamics [14], and weakly coupled layered systems [15], [16].

Due to the presence of band gaps in periodic media, many researchers have been focusing on wave propagation in nonlinear periodic structures and its application to the design of novel metamaterials [3], [9]-[11], [17]-[20]. Structures exhibiting band gaps prevent the propagation of waves at certain frequencies. These structures may be phononic (sonic) or photonic, depending on their band-gap frequency range. Phononic or sonic band-gap structures can be used as sensing devices based on resonators, acoustic logic ports and wave guides, and frequency filters based on surface acoustic waves, while photonic band-gap structures have applications in optics and microwaves. Synthesis of phononic materials with desired band-gap and wave-guiding characteristics has been achieved through the application of topology and material optimization procedures [7], [21], [22]. The application of periodic plane grid structures as phononic materials and its design optimization process has been presented in [23], where a limited number of continuously varying parameters define the geometry of a predefined cellular topology that deals with periodic structures of infinite size as well as demonstrate the validity of the results to finite systems.

Amplitude-dependent dispersion and band-gap behavior have been explored in several discrete periodic systems characterized by cubic nonlinearities by Narisetti et al. [10], where it was shown that the boundary of the dispersion curve may shift with amplitude in the presence of a single plane wave. Manktelow et al. [24] have recently extended the analysis in [10] to include the propagation of multiple harmonic plane waves that show the dispersion properties of discrete, periodic, cubically nonlinear systems. They presented a comparison between the multiple scales and Lindstedt-Poincare method for harmonic wave-wave interactions due to commensurate frequency ratios. Narisetti et al. [11] have recently studied a plane wave propagation in strongly nonlinear periodic media. At higher frequencies and low amplitudes, it is shown that a hexagonally-packed and prestressed lattice exhibits acoustic wave beaming phenomena and may have applications in tunable spatial filters and tunable stress-redirecting materials.

*corresponding author: Scientist/Research Engineer at Ford Motor Company, Research \& Advanced Engineering. email: smrutiranjan@gmail.com. 
While these studies address cubic nonlinear periodic media for applications as metamaterials, there has been less attention on wave propagations in periodic media with quadratic nonlinearities. This study is motivated by the snap-through waves that are expected to exist in chains of bistable mass-spring elements. In [25], [26], we studied the bifurcations of equilibria with respect to a quasistatic pull, for a two-degree-of-freedom snap-through oscillator connected by bistable springs. Here we look at small amplitude waves propagating through a nonlinear chain that retains the quadratic and cubic nonlinearity local to the equilibrium, for example in a snap-through chain. A second-order multiple scales perturbation analysis is applied for low-amplitude oscillations that capture the quadratic effects. We obtain a nonlinear dispersion relationship from the theoretical analysis and compare it to linear and cubically nonlinear cases. The amplitude dependence of the dispersion relations shows that the mass-spring chains can be used as tunable acoustic filters. The group and phase velocity dependence on wave number and amplitude shows the relevance of quadratic effects for applications in band gaps, event detection and nonlinear waveguides.

\section{Periodic Chain of Nonlinear Oscillators}

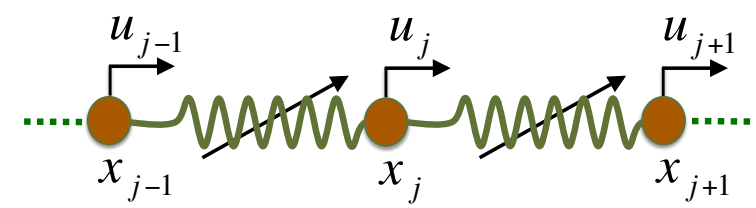

Fig. 1: Infinite mass chain. The unstretched position and displacement of mass $m_{j}$ are denoted by $x_{j}$ and $u_{j}$ respectively. The springs have unstretched length $h$ and are cubic nonlinear with characteristics shown in Figure 2.

The mass-spring chain is arranged in a fashion such that each mass is separated by a distance $h$ from its nearest neighbor at equilibrium. We use the assumption that all the masses are equal $\left(m_{j}=m\right)$ and only the nearest neighbors have direct effects on each other. The nonlinear spring characteristics illustrated in Figure 2 with both the quadratic and cubic nonlinearity is given by

$$
f\left(s_{j}\right)=\alpha s_{j}+\beta s_{j}^{2}+\gamma s_{j}^{3}
$$

where $s_{j}$ is the stretch or the deformation of the $j^{\text {th }}$ spring.

As such, we consider a cubic expansion of the spring force relative to the equilibrium state. The equations of motion (EOM) in physical coordinate can then be written as

$$
m \ddot{\tilde{u}}_{j}=\tilde{\alpha}\left[\left(\tilde{u}_{j+1}-\tilde{u}_{j}\right)-\left(\tilde{u}_{j}-\tilde{u}_{j-1}\right)\right]+\tilde{\beta}\left[\left(\tilde{u}_{j+1}-\tilde{u}_{j}\right)^{2}-\left(\tilde{u}_{j}-\tilde{u}_{j-1}\right)^{2}\right]+\tilde{\gamma}\left[\left(\tilde{u}_{j+1}-\tilde{u}_{j}\right)^{3}-\left(\tilde{u}_{j}-\tilde{u}_{j-1}\right)^{3}\right]
$$

for $j=\cdots,-2,-1,0,1,2, \cdots$. For small oscillations we assume $\tilde{u}=\epsilon u$ and then simplify the equation by letting $\alpha=\frac{\tilde{\alpha}}{m}$, $\beta=\frac{\tilde{\beta}}{m}$, and $\gamma=\frac{\tilde{\gamma}}{m}$. We apply the following coordinate transformation

For small amplitude of oscillations we assume

$$
\tilde{u}_{j}=\epsilon u_{j}
$$

where $u_{j}$ is of $O(1)$ and $\tilde{u}_{j}$ is of $O(\epsilon)$. Letting $\alpha=\frac{\tilde{\alpha}}{m}, \beta=\frac{\tilde{\beta}}{m}$, and $\gamma=\frac{\tilde{\gamma}}{m}$, this transformation results in the undamped nonlinear equation of motion of the $j^{\text {th }}$ mass for weakly nonlinear interactions with the adjacent elements as bellow,

$$
\ddot{u}_{j}=\alpha\left[\left(u_{j+1}-u_{j}\right)-\left(u_{j}-u_{j-1}\right)\right]+\epsilon \beta\left[\left(u_{j+1}-u_{j}\right)^{2}-\left(u_{j}-u_{j-1}\right)^{2}\right]+\epsilon^{2} \gamma\left[\left(u_{j+1}-u_{j}\right)^{3}-\left(u_{j}-u_{j-1}\right)^{3}\right]
$$

for $j=\cdots,-2,-1,0,1,2, \cdots$. To simplify the equation we apply the following coordinate transformation:

$$
z_{j}=\left(u_{j+1}-u_{j}\right)
$$

to get the following equations

$$
\begin{gathered}
\ddot{u}_{j+1}=\alpha\left(z_{j+1}-z_{j}\right)+\epsilon \beta\left(z_{j+1}^{2}-z_{j}^{2}\right)+\epsilon^{2} \gamma\left(z_{j+1}^{3}-z_{j}^{3}\right) \\
\ddot{u}_{j}=\alpha\left(z_{j}-z_{j-1}\right)+\epsilon \beta\left(z_{j}^{2}-z_{j-1}^{2}\right)+\epsilon^{2} \gamma\left(z_{j}^{3}-z_{j-1}^{3}\right)
\end{gathered}
$$

where $z_{j}$ is the strain in the $j^{\text {th }}$ spring. Now subtracting equation (7) from equation (6), we obtain the equation of motion in the $z$ coordinates,

$$
\ddot{z}_{j}=-\alpha\left(2 z_{j}-z_{j+1}-z_{j-1}\right)-\epsilon \beta\left(2 z_{j}^{2}-z_{j+1}^{2}-z_{j-1}^{2}\right)-\epsilon^{2} \gamma\left(2 z_{j}^{3}-z_{j+1}^{3}-z_{j-1}^{3}\right)
$$

When $\epsilon$ is small, we are considering the spring deformation near one of the stable equilibria, i.e. it does not snap through. With $\epsilon$ as a bookkeeping parameter, the quadratic effect is more significant than the cubic in equation (8). With the small parameter $\epsilon$, we will analyze these ordinary differential equations by using the method of multiple scales next. 

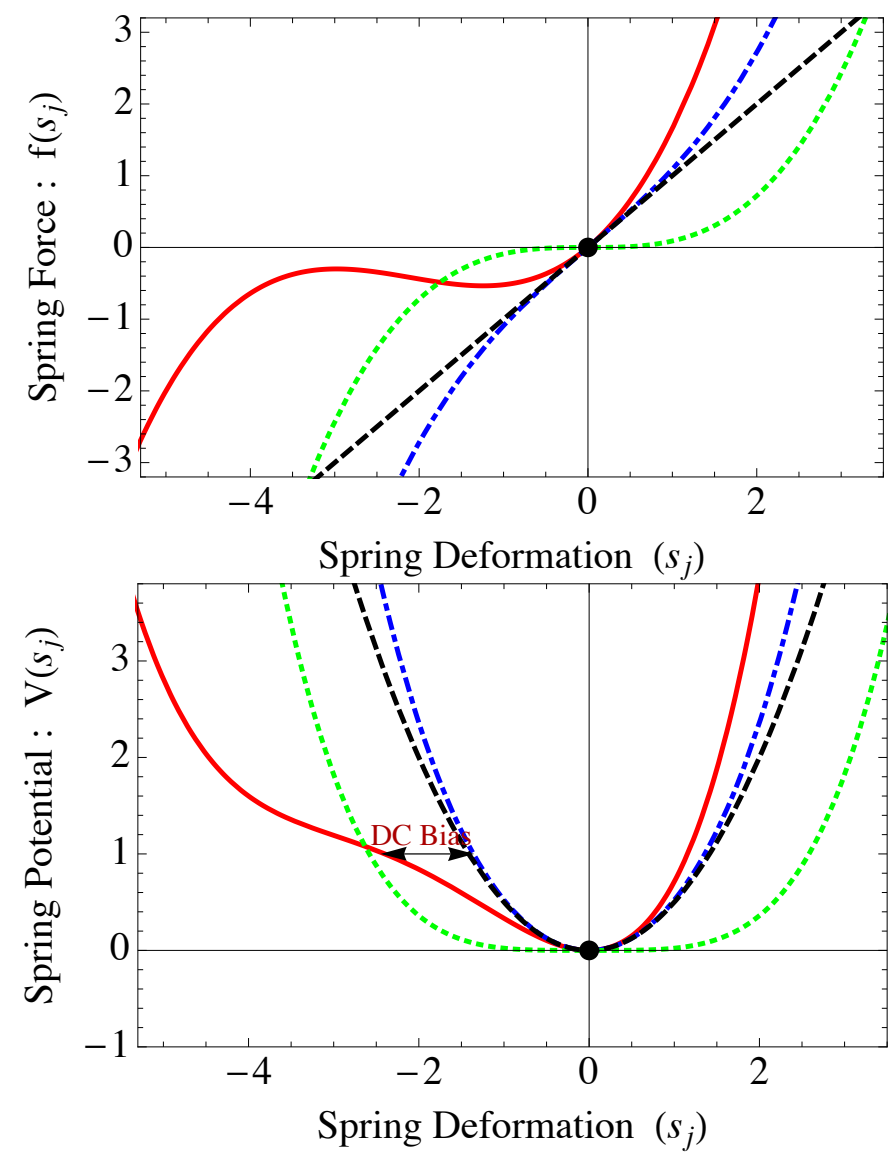

Fig. 2: The characteristic spring force $f\left(s_{j}\right)$, and spring potential $V\left(s_{j}\right)$ of the linear and weakly nonlinear spring as a function of the spring deformation $s_{j}$. Referring to the spring force with characteristics as in equation (1), the solid (red) curve corresponds to $\alpha=1, \beta=0.57$, and $\gamma=0.09$, Dashed (black) line corresponds to $\alpha=1, \beta=0$, and $\gamma=0$, dot-dashed (blue) curve correspond to $\alpha=1, \beta=0$, and $\gamma=0.09$, and the dotted (green) curve correspond to $\alpha=0, \beta=0$, and $\gamma=0.09$. The potential well shown to be shifted to the left with positive quadratic nonlinearity, hence indicates a negative DC-bias.

\section{WaVe Dispersion Analysis Using Second-Order Multiple Scales}

\section{A. Traveling Wave Solutions in Strain Coordinates}

We analyze the wave equation using the second-order method of multiple scales (MMS). We assume,

$$
\begin{aligned}
& z_{j}(t)=z_{j_{0}}\left(T_{0}, T_{1}, T_{2}\right)+\epsilon z_{j_{1}}\left(T_{0}, T_{1}, T_{2}\right)+\epsilon^{2} z_{j_{2}}\left(T_{0}, T_{1}, T_{2}\right)+\cdots \\
& \frac{d}{d t}=D_{0}+\epsilon D_{1}+\epsilon^{2} D_{2}+\cdots \\
& \frac{d^{2}}{d t}=D_{0}^{2}+\epsilon\left(2 D_{0} D_{1}\right)+\epsilon^{2}\left(D_{1}^{2}+2 D_{0} D_{2}\right)+\cdots
\end{aligned}
$$

where $D_{i}=\frac{\partial}{\partial T_{i}}$. Substituting equation (9) into equation (8) yields

$$
\begin{aligned}
D_{0}^{2} z_{j_{0}}+\epsilon\left[D_{0}^{2} z_{j_{1}}\right. & \left.+2 D_{0} D_{1} z_{j_{0}}\right]+\epsilon^{2}\left[D_{0}^{2} z_{j_{2}}+2 D_{0} D_{1} z_{j_{1}}+\left(D_{1}^{2}+2 D_{0} D_{2}\right) z_{j_{0}}\right]= \\
& -\alpha\left[2 z_{j_{0}}-z_{j+1_{0}}-z_{j-1_{0}}\right]-\epsilon\left[\alpha\left(2 z_{j_{1}}-z_{j+1_{1}}-z_{j-1_{1}}\right)+\beta\left(2 z_{j_{0}}^{2}-z_{j+1_{0}}^{2}-z_{j-1_{0}}^{2}\right)\right] \\
& -\epsilon^{2}\left[\alpha\left(2 z_{j_{2}}-z_{j+1_{2}}-z_{j-1_{2}}\right)+\gamma\left(2 z_{j_{0}}^{3}-z_{j+1_{0}}^{3}-z_{j-1_{0}}^{3}\right)+2 \beta\left(2 z_{j_{0}} z_{j_{1}}-z_{j+1_{0}} z_{j+1_{1}}-z_{j-1_{0}} z_{j-1_{1}}\right)\right]
\end{aligned}
$$


Equating the like powers of $\epsilon$,

$$
\begin{aligned}
\epsilon^{0}: D_{0}^{2} z_{j_{0}}+\alpha\left(2 z_{j_{0}}-z_{j+1_{0}}-z_{j-1_{0}}\right)= & 0 \\
\epsilon^{1}: D_{0}^{2} z_{j_{1}}+\alpha\left(2 z_{j_{1}}-z_{j+1_{1}}-z_{j-1_{1}}\right)= & -2 D_{0} D_{1} z_{j_{0}}-\beta\left(2 z_{j_{0}}^{2}-z_{j+1_{0}}^{2}-z_{j-1_{0}}^{2}\right) \\
\epsilon^{2}: D_{0}^{2} z_{j_{2}}+\alpha\left(2 z_{j_{2}}-z_{j+1_{2}}-z_{j-1_{2}}\right)= & -2 D_{0} D_{1} z_{j_{1}}-\left(D_{1}^{2}+2 D_{0} D_{2}\right) z_{j_{0}} \\
& -2 \beta\left(2 z_{j_{0}} z_{j_{1}}-z_{j+1_{0}} z_{j+1_{1}}-z_{j-1_{0}} z_{j-1_{1}}\right)-\gamma\left(2 z_{j_{0}}^{3}-z_{j+1_{0}}^{3}-z_{j-1_{0}}^{3}\right)
\end{aligned}
$$

Let us assume a traveling dispersive wave solution to solve for the $\epsilon^{0}$ equation in the above equation-set (11). Let

$$
z_{j_{0}}=y_{j_{0}}+\bar{y}_{j_{0}}=A e^{i\left(k x_{j}-\omega_{0} T_{0}\right)}+\bar{A} e^{-i\left(k x_{j}-\omega_{0} T_{0}\right)}
$$

where $y_{j_{0}}=A e^{i\left(k x_{j}-\omega_{0} T_{0}\right)}$. Then $y_{j \pm 1_{0}}=A e^{i\left(k x_{j \pm 1}-\omega_{0} T_{0}\right)}$. We also assume $x_{j \pm 1}=x_{j} \pm h$. We use $y_{j \pm 1_{0}}=e^{ \pm i k h} A e^{i\left(k x_{j}-\omega_{0} T_{0}\right)}=$ $e^{ \pm i k h} y_{j_{0}}$, into the $\epsilon^{0}$ equation in equations (11) we get

$$
\omega_{0}^{2}=2 \alpha(1-\cos k h)
$$

Also, since $A$ can be complex, we express $A$ in terms of real amplitude and phase as $A=\frac{1}{2} a e^{-i \theta}$, such that

$$
z_{j_{0}}=y_{j_{0}}+\bar{y}_{j_{0}}=\frac{1}{2} a e^{i\left(k x_{j}-\omega_{0} T_{0}-\theta\right)}+c . c .
$$

The solution to $\epsilon^{0}$ equation can be used in the $\epsilon^{1}$ equation from equation-set (11) to find the resonant terms that contribute to secularity or unbounded solutions. The resonant terms are those associate with $e^{i\left(k x_{j}-\omega_{0} T_{0}\right)}$. The solvability condition resulting from the secularity is $-2 D_{0} D_{1} z_{j_{0}}=2\left(i \omega_{0}\right) A^{\prime} e^{i\left(k x_{j}-\omega_{0} T_{0}\right)}+c . c$. Hence $A^{\prime}=0$, where ()$^{\prime}=\frac{\partial}{\partial T_{1}}$, i.e. $a^{\prime}=0$ and $\theta^{\prime}=0$, such that

$$
\begin{aligned}
& a=a\left(T_{2}\right) \\
& \theta=\theta\left(T_{2}\right)
\end{aligned}
$$

Removing the secular term i.e. coefficients of $e^{i\left(k x_{j}-\omega_{0} T_{0}\right)}$, we write the $\epsilon^{1}$ equation as

$$
\begin{aligned}
D_{0}^{2} z_{j_{1}}+\alpha\left(2 z_{j_{1}}-z_{j+1_{1}}-z_{j-1_{1}}\right) & =-\beta\left(2 z_{j_{0}}^{2}-z_{j+1_{0}}^{2}-z_{j-1_{0}}^{2}\right) \\
& =-\beta\left(2-e^{2 i k h}-e^{-2 i k h}\right)\left(\frac{1}{2} a e^{-i \theta}\right)^{2} e^{2 i\left(k x_{j}-\omega_{0} T_{0}\right)}+c . c . \\
& =-\frac{\beta a^{2}}{2}(1-\cos 2 k h) e^{2 i\left(k x_{j}-\omega_{0} T_{0}-\theta\right)}+\text { c.c. }
\end{aligned}
$$

The particular solution to the above equation will therefore be of the form,

$$
z_{j_{1}}=A_{1} e^{2 i\left(k x_{j}-\omega_{0} T_{0}-\theta\right)}+\text { c.c. }
$$

In order to find $A_{1}$ in terms of $A$, we substitute the assumed solution $z_{1}$ into the $\epsilon^{1}$ equation. Balancing the coefficients of $e^{2 i\left(k x_{j}-\omega_{0} T_{0}-\theta\right)}$ leads to

$$
A_{1}=\frac{\beta a^{2} \sin ^{2} k h}{4\left(\omega_{0}^{2}-\alpha \sin ^{2} k h\right)}
$$

The order- $\epsilon$ solution $z_{j_{1}}$ is therefore

$$
z_{j_{1}}=\frac{\beta a^{2} \sin ^{2} k h}{4\left(\omega_{0}^{2}-\alpha \sin ^{2} k h\right)} e^{2 i\left(k x_{j}-\omega_{0} T_{0}-\theta\right)}+c . c .
$$

To solve for the amplitudes $a$ and phase $\theta$, we examine the $\epsilon^{2}$ equation. We note that $z_{j_{0}}$ and $z_{j_{1}}$ are independent of slow time-scale $T_{1}$, since $A$ is independent of $T_{1}$. Therefore $\left(-2 D_{0} D_{1} z_{j_{1}}\right)$ and $\left(D_{1}^{2} z_{j_{0}}\right)$ vanish in the $\epsilon^{2}$ equation. Using the solutions of $z_{j_{0}}$ and $z_{j_{1}}$, we obtain the various terms of the right hand side of the $\epsilon^{2}$ equation of the equation-set (11), as below:

$$
\begin{aligned}
2 D_{0} D_{2} z_{j_{0}} & =\left(a^{\prime}-a i b^{\prime}\right)\left(-i \omega_{0}\right) e^{i\left(k x_{j}-\omega_{0} T_{0}-\theta\right)}+c . c . \\
z_{j_{0}} z_{j_{1}} & =\frac{\beta a^{3} \sin ^{2} k h}{8\left(\omega_{0}^{2}-\alpha \sin ^{2} k h\right)}\left[e^{3 i\left(k x_{j}-\omega_{0} T_{0}-\theta\right)}+e^{i\left(k x_{j}-\omega_{0} T_{0}-\theta\right)}\right]+c . c . \\
z_{j \pm 1_{0}} z_{j \pm 1_{1}} & =\frac{\beta a^{3} \sin ^{2} k h}{8\left(\omega_{0}^{2}-\alpha \sin ^{2} k h\right)}\left[e^{ \pm 3 i k h} e^{3 i\left(k x_{j}-\omega_{0} T_{0}-\theta\right)}+e^{ \pm i k h} e^{i\left(k x_{j}-\omega_{0} T_{0}-\theta\right)}\right]+c . c . \\
z_{j_{0}}^{3} & =\frac{a^{3}}{8}\left[e^{3 i\left(k x_{j}-\omega_{0} T_{0}-\theta\right)}+3 e^{i\left(k x_{j}-\omega_{0} T_{0}-\theta\right)}\right]+c . c . \\
z_{j \pm 1_{0}}^{3} & =\frac{a^{3}}{8}\left[e^{ \pm 3 i k h} e^{3 i\left(k x_{j}-\omega_{0} T_{0}-\theta\right)}+3 e^{ \pm i k h} e^{i\left(k x_{j}-\omega_{0} T_{0}-\theta\right)}\right]+c . c .
\end{aligned}
$$


where, $a^{\prime}=\frac{\partial a}{\partial T_{2}}$, and $\theta^{\prime}=\frac{\partial \theta}{\partial T_{2}}$. Using the above simplifications in the $\epsilon^{2}$ equation, we get

$$
\begin{aligned}
D_{0}^{2} z_{j_{2}} & +\alpha\left(2 z_{j_{2}}-z_{j+1_{2}}-z_{j-1_{2}}\right)=-\left[\left(a^{\prime}-a i \theta^{\prime}\right)\left(-i \omega_{0}\right) e^{i\left(k x_{j}-\omega_{0} T_{0}-\theta\right)}+c . c .\right] \\
& -2 \beta \frac{\beta a^{3} \sin ^{2} k h}{8\left(\omega_{0}^{2}-\alpha \sin ^{2} k h\right)}\left[\left(2-e^{3 i k h}-e^{-3 i k h}\right) e^{3 i\left(k x_{j}-\omega_{0} T_{0}-\theta\right)}+c . c .+\left(2-e^{i k h}-e^{-i k h}\right) e^{i\left(k x_{j}-\omega_{0} T_{0}-\theta\right)}+c . c .\right] \\
& -\gamma \frac{a^{3}}{8}\left[\left(2-e^{3 i k h}-e^{-3 i k h}\right) e^{3 i\left(k x_{j}-\omega_{0} T_{0}-\theta\right)}+\text { c.c. }+3\left(2-e^{i k h}-e^{-i k h}\right) e^{i\left(k x_{j}-\omega_{0} T_{0}-\theta\right)}+\text { c.c. }\right]
\end{aligned}
$$

where, $a^{\prime}=\frac{\partial a}{\partial T_{2}}$, and $\theta^{\prime}=\frac{\partial b}{\partial T_{2}}$. The secular terms from the above equation are the coefficients of $e^{i\left(k x_{j}-\omega_{0} T_{0}\right)}$. Eliminating the secular term results in

$$
\left(i \omega_{0} a^{\prime}+\omega_{0} a \theta^{\prime}\right)=\left[\frac{3 \gamma}{4}+\frac{\beta^{2} \sin ^{2} k h}{2\left(\omega_{0}^{2}-\alpha \sin ^{2} k h\right)}\right](1-\cos k h) a^{3}
$$

Equating the real and imaginary parts, the solvability condition can be written as

$$
\begin{array}{ll}
\text { Im: } & a^{\prime}=0 \\
\text { Re: } & \omega_{0} a \theta^{\prime}=\left[\frac{3 \gamma}{4}+\frac{\beta^{2} \sin ^{2} k h}{2\left(\omega_{0}^{2}-\alpha \sin ^{2} k h\right)}\right](1-\cos k h) a^{3}
\end{array}
$$

Using $\omega_{0}$ from equation (13), in equation (23), we get

$$
\begin{array}{ll}
\text { Im: } & a^{\prime}=0 \\
\text { Re: } & \theta^{\prime}=\left[\gamma+\frac{2 \beta^{2}(1+\cos k h)}{3 \alpha(1-\cos k h)}\right] \frac{3 \omega_{0}}{8 \alpha} a^{2}
\end{array}
$$

Solving the equation (24), we get

$$
\begin{aligned}
& a=a_{0} \\
& \theta=\left[\gamma+\frac{2 \beta^{2}(1+\cos k h)}{3 \alpha(1-\cos k h)}\right] \frac{3 \omega_{0}}{8 \alpha} a_{0}^{2} T_{2}+\theta_{c}
\end{aligned}
$$

We will neglect the integration constant $\theta_{c}$ without loss of generality. Also note that $T_{2}=\epsilon^{2} T_{0}$. Hence the slowly varying amplitude $(a)$ and phase $(\theta)$ becomes,

$$
\begin{aligned}
a & =a_{0} \\
\theta & =\left[\gamma+\frac{2 \beta^{2}(1+\cos k h)}{3 \alpha(1-\cos k h)}\right] \frac{3 \omega_{0}}{8 \alpha} a_{0}^{2} \epsilon^{2} T_{0}
\end{aligned}
$$

Therefore, substituting $\theta$ from equation (26) into the second-order approximation of the frequency, $\omega$, derived from equation (14) using $\omega t=\omega T_{0}=\omega_{0} T_{0}+\theta$, the frequency $\omega$ can be written as

$$
\omega=\omega_{0}+\frac{\theta}{T_{0}}=\omega_{0}\left[1+\frac{3 \epsilon^{2} a_{0}^{2}}{8 \alpha}\left(\gamma+\frac{2 \beta^{2}(1+\cos k h)}{3 \alpha(1-\cos k h)}\right)\right]
$$

\section{B. Frequency Expression in Displacement Coordinates}

The above solution in equation (27) is obtained by solving the EOM in strain coordinates $z_{j}=u_{j+1}-u_{j}$, where $u_{j}$ is in the displacement coordinates. We now interprete the solution in displacement coordinates. Let the solution in $u$ coordinates be $u_{j}=D e^{i\left(k x_{j}-\omega t\right)}+c . c$. and $u_{j+1}=D e^{i\left(k x_{j+1}-\omega t\right)}+c . c .=D e^{i k h} e^{i\left(k x_{j}-\omega t\right)}+c . c$. Therefore

$$
z_{j}=D\left(e^{i k h}-1\right) e^{i\left(k x_{j}-\omega t\right)}+c . c .=A e^{i\left(k x_{j}-\omega t\right)}+c . c .
$$

Letting $D=\frac{1}{2} d_{0} e^{-i \hat{\theta}}$, we get the relationship between the amplitudes in displacement and strain coordinates as

$$
\begin{aligned}
& A=\frac{1}{2} d_{0} e^{-i \hat{\theta}}\left(e^{i k h}-1\right)=\frac{1}{2} d_{0} e^{-i \hat{\theta}}\left(\rho e^{i \phi}\right) \\
& \text { or } \quad \frac{1}{2} a_{0} e^{-i \theta}=\frac{1}{2} \rho d_{0} e^{-i(\hat{\theta}-\phi)}
\end{aligned}
$$


where, with help from equation (13),

$$
\begin{aligned}
& \rho=\sqrt{(\cos k h-1)^{2}+\sin ^{2} k h}=\sqrt{2(1-\cos k h)}=\sqrt{\frac{\omega_{0}^{2}}{\alpha}} \\
& \tan \phi=\frac{\sin k h}{\cos k h-1}=-\cot \frac{k h}{2}=\tan \left(\frac{\pi}{2}+\frac{k h}{2}\right) \\
& a_{0}=\rho d_{0}
\end{aligned}
$$

Hence the amplitude and phase in strain coordinates are related to the amplitude and phase in displacement coordinates by

$$
\begin{aligned}
& a_{0}^{2}=\frac{\omega_{0}^{2}}{\alpha} d_{0}^{2} \\
& \theta=\hat{\theta}-\left(\frac{\pi}{2}+\frac{k h}{2}\right)
\end{aligned}
$$

Therefore the frequency in the displacement coordinate system can be written as

$$
\omega\left(k, d_{0}\right)=\omega_{0}\left[1+\epsilon^{2}\left\{\gamma+\frac{2 \beta^{2}}{3 \alpha}\left(\frac{1+\cos k h}{1-\cos k h}\right)\right\} \frac{3 \omega_{0}^{2} d_{0}^{2}}{8 \alpha^{2}}\right]
$$

Also note that, in the above dispersion relationship, $\omega_{0}$ is a function of the wave number $k$ as in equation (13).

\section{Comparison in the Continuum Limit}

To check the correlation between the coordinates, we consider a continuum. The displacement at any location $x$ in the continuum is assumed to be of the following form

$$
u\left(x_{j}\right)=D e^{i\left(k x_{j}-\omega t\right)}
$$

The strain can then be found by taking the partial derivative of the displacement with respect to position $x$ to get

$$
z\left(x_{j}, t\right)=\frac{\partial u\left(x_{j}\right)}{\partial x_{j}}=D(i k) e^{i\left(k x_{j}-\omega t\right)}=D k e^{i \frac{\pi}{2}} e^{i\left(k x_{j}-\omega t\right)}
$$

To obtain the continuum limit in our MMS solution, we first represent the amplitude and phase of our solution (strain) in terms of displacement coordinates and then take the limit as $h \rightarrow 0$. Making use of

$$
\lim _{h \rightarrow 0} \frac{\sin (k h)}{h}=k
$$

and the equations (28) - (30) the strain can be found as

$$
\begin{aligned}
z\left(x_{j}, t\right) & =\lim _{h \rightarrow 0} \frac{z_{j}\left(x_{j}, t\right)}{h}=\lim _{h \rightarrow 0} A \frac{1}{h} e^{i\left(k x_{j}-\omega t\right)} \\
& =\lim _{h \rightarrow 0} D \frac{1}{h}\left(e^{i k h}-1\right) e^{i\left(k x_{j}-\omega t\right)}=\lim _{h \rightarrow 0} D \rho \frac{1}{h} e^{i \phi} e^{i\left(k x_{j}-\omega t\right)} \\
& =\lim _{h \rightarrow 0} D \frac{2 \sin \left(\frac{k h}{2}\right)}{h} e^{i\left(\frac{\pi}{2}+\frac{k h}{2}\right)} e^{i\left(k x_{j}-\omega t\right)}=D k e^{i \frac{\pi}{2}} e^{i\left(k x_{j}-\omega t\right)}
\end{aligned}
$$

Hence the strains from both equations (34) and (36) are identical and confirm the validity of the coordinate transformations between the strain and displacement coordinates.

\section{Alternate Method: Single Wave Dispersion Analysis in the ContinuUm Limit}

\section{A. Partial Differential Equations for Dispersion Analysis}

The Taylor series expansion of $\tilde{u}_{j \pm 1}(\tilde{x})$ about $\tilde{u}_{j}(\tilde{x})$ is

$$
\begin{aligned}
& \tilde{u}_{j+1}=\tilde{u}_{j}+\frac{\partial \tilde{u}_{j}}{\partial \tilde{x}} h+\frac{\partial^{2} \tilde{u}_{j}}{2 ! \partial \tilde{x}^{2}} h^{2}+\frac{\partial^{3} \tilde{u}_{j}}{3 ! \partial \tilde{x}^{3}} h^{3}+\frac{\partial^{4} \tilde{u}_{j}}{4 ! \partial \tilde{x}^{4}} h^{4}+\cdots \\
& \tilde{u}_{j-1}=\tilde{u}_{j}-\frac{\partial \tilde{u}_{j}}{\partial \tilde{x}} h+\frac{\partial^{2} u_{j}}{2 ! \partial \tilde{x}^{2}} h^{2}-\frac{\partial^{3} \tilde{u}_{j}}{3 ! \partial \tilde{x}^{3}} h^{3}+\frac{\partial^{4} \tilde{u}_{j}}{4 ! \partial \tilde{x}^{4}} h^{4}+\cdots
\end{aligned}
$$


for $j=\cdots,-2,-1,0,1,2, \cdots$. We denote $\tilde{u}_{t t}=\frac{\partial^{2} \tilde{u}_{j}}{\partial t^{2}}=\ddot{\tilde{u}}_{j}, \tilde{u}_{\tilde{x}}=\frac{\partial \tilde{u}_{j}}{\partial \tilde{x}}, \tilde{u}_{\tilde{x} \tilde{x}}=\frac{\partial^{2} \tilde{u}_{j}}{\partial \tilde{x}^{2}}, \cdots, \tilde{u}_{\tilde{x}^{\tilde{x} \text {-times }} \ldots \tilde{x}}^{\tilde{x}}=\frac{\partial^{n} \tilde{u}_{j}}{\partial \tilde{x}^{n}}$ in equation $(37)$, and letting $\alpha=\frac{\tilde{\alpha}}{m_{j}}, \beta=\frac{\tilde{\beta}}{m_{j}}, \gamma=\frac{\tilde{\gamma}}{m_{j}}$, equation (4) is transformed as

$$
\begin{aligned}
& \tilde{u}_{t t} \approx \alpha \tilde{u}_{\tilde{x} \tilde{x}} h^{2}+2 \beta \tilde{u}_{\tilde{x}} \tilde{u}_{\tilde{x} \tilde{x}} h^{3}+\left(\frac{\alpha}{12} \tilde{u}_{\tilde{x} \tilde{x} \tilde{x} \tilde{x}}+3 \gamma \tilde{u}_{\tilde{x}}^{2} \tilde{u}_{\tilde{x} \tilde{x}}\right) h^{4}+\left(\frac{\beta}{6} \tilde{u}_{\tilde{x}} \tilde{u}_{\tilde{x} \tilde{x} \tilde{x} \tilde{x}}+\frac{\beta}{3} \tilde{u}_{\tilde{x} \tilde{x}} \tilde{u}_{\tilde{x} \tilde{x} \tilde{x}}\right) h^{5} \\
& +\left(\frac{\alpha}{360} \tilde{u}_{\tilde{x} \tilde{x} \tilde{x} \tilde{x} \tilde{x}}+\frac{\gamma}{4} \tilde{u}_{\tilde{x}}^{2} \tilde{u}_{\tilde{x} \tilde{x} \tilde{x} \tilde{x}}+\gamma \tilde{u}_{\tilde{x}} \tilde{u}_{\tilde{x} \tilde{x}} \tilde{u}_{\tilde{x} \tilde{x} \tilde{x}}+\frac{\gamma}{4} \tilde{u}_{\tilde{x} \tilde{x}}^{3}\right) h^{6}+\left(\frac{\beta}{180} \tilde{u}_{\tilde{x}} \tilde{u}_{\tilde{x} \tilde{x} \tilde{x} \tilde{x} \tilde{x} \tilde{x}}+\frac{\beta}{60} \tilde{u}_{\tilde{x} \tilde{x}} \tilde{u}_{\tilde{x} \tilde{x} \tilde{x} \tilde{x} \tilde{x}}+\frac{\beta}{36} \tilde{u}_{\tilde{x} \tilde{x} \tilde{x}} \tilde{u}_{\tilde{x} \tilde{x} \tilde{x} \tilde{x}}\right) h^{7} \\
& +\left(\frac{\alpha}{20160} \tilde{u}_{\tilde{x} \tilde{x} \tilde{x} \tilde{x} \tilde{x} \tilde{x} \tilde{x}}+\frac{\gamma}{120} \tilde{u}_{\tilde{x}}^{2} \tilde{u}_{\tilde{x} \tilde{x} \tilde{x} \tilde{x} \tilde{x} \tilde{x}}+\frac{\gamma}{20} \tilde{u}_{\tilde{x}} \tilde{u}_{\tilde{x} \tilde{x}} \tilde{u}_{\tilde{x} \tilde{x} \tilde{x} \tilde{x} \tilde{x}}+\frac{\gamma}{12} \tilde{u}_{\tilde{x}} \tilde{u}_{\tilde{x} \tilde{x} \tilde{x}} \tilde{u}_{\tilde{x} \tilde{x} \tilde{x} \tilde{x}}+\frac{\gamma}{12} \tilde{u}_{\tilde{x} \tilde{x}} \tilde{u}_{\tilde{x} \tilde{x} \tilde{x}}^{2}+\frac{\gamma}{16} \tilde{u}_{\tilde{x} \tilde{x}}^{2} \tilde{u}_{\tilde{x} \tilde{x} \tilde{x} \tilde{x}}\right) h^{8}
\end{aligned}
$$

Nondimensionalizing the above equation by letting $x=\frac{\tilde{x}}{h}$, then $\frac{\partial^{n}}{\partial \tilde{x}^{n}}=\frac{1}{h^{n}} \frac{\partial^{n}}{\partial x^{n}}$ the following partial differential equation (PDE) is obtained.

$$
\begin{aligned}
\tilde{u}_{t t} \approx & \alpha \tilde{u}_{x x}+2 \beta \tilde{u}_{x} \tilde{u}_{x x}+\left(\frac{\alpha}{12} \tilde{u}_{x x x x}+3 \gamma \tilde{u}_{x}^{2} \tilde{u}_{x x}\right)+\left(\frac{\beta}{6} \tilde{u}_{x} \tilde{u}_{x x x x}+\frac{\beta}{3} \tilde{u}_{x x} \tilde{u}_{x x x}\right) \\
& +\left(\frac{\alpha}{360} \tilde{u}_{x x x x x x}+\frac{\gamma}{4} \tilde{u}_{x}^{2} \tilde{u}_{x x x x}+\gamma \tilde{u}_{x} \tilde{u}_{x x} \tilde{u}_{x x x}+\frac{\gamma}{4} \tilde{u}_{x x}^{3}\right)+\left(\frac{\beta}{180} \tilde{u}_{x} \tilde{u}_{x x x x x}+\frac{\beta}{60} \tilde{u}_{x x} \tilde{u}_{x x x x x}+\frac{\beta}{36} \tilde{u}_{x x x} \tilde{u}_{x x x x}\right) \\
& +\left(\frac{\alpha}{20160} \tilde{u}_{x x x x x x x x}+\frac{\gamma}{120} \tilde{u}_{x}^{2} \tilde{u}_{x x x x x x}+\frac{\gamma}{20} \tilde{u}_{x} \tilde{u}_{x x} \tilde{u}_{x x x x x}+\frac{\gamma}{12} \tilde{u}_{x} \tilde{u}_{x x x} \tilde{u}_{x x x x}+\frac{\gamma}{12} \tilde{u}_{x x} \tilde{u}_{x x x}^{2}+\frac{\gamma}{16} \tilde{u}_{x x}^{2} \tilde{u}_{x x x x}\right)
\end{aligned}
$$

For small oscillations with the spring deformation near one of the stable equilibria, we let $\tilde{u}=\epsilon u, \tilde{u}_{t t}=\epsilon u_{t t}, \tilde{u}_{x \ldots x}=\epsilon u_{x \ldots x}$, etc., in equation (39) and cancel $\epsilon$ from both sides, to obtain

$$
\begin{aligned}
u_{t t} \approx & \alpha\left(\frac{u_{x x}}{2 !}+\frac{u_{x x x x}}{4 !}+\frac{u_{x x x x x x}}{6 !}+\frac{u_{x x x x x x x x}}{8 !}\right) \\
& +\epsilon\left[2 \beta u_{x} u_{x x}+\left(\frac{\beta}{6} u_{x} u_{x x x x}+\frac{\beta}{3} u_{x x} u_{x x x}\right)+\left(\frac{\beta}{180} u_{x} u_{x x x x x x}+\frac{\beta}{60} u_{x x} u_{x x x x x}+\frac{\beta}{36} u_{x x x} u_{x x x x}\right)\right] \\
& +\epsilon^{2}\left[3 \gamma u_{x}^{2} u_{x x}+\left(\frac{\gamma}{4} u_{x}^{2} u_{x x x x}+\gamma u_{x} u_{x x} u_{x x x}+\frac{\gamma}{4} u_{x x}^{3}\right)+\left(\frac{\gamma}{120} u_{x}^{2} u_{x x x x x x}+\frac{\gamma}{20} u_{x} u_{x x} u_{x x x x x}\right.\right. \\
& \left.\left.+\frac{\gamma}{12} u_{x} u_{x x x} u_{x x x x}+\frac{\gamma}{12} u_{x x} u_{x x x}^{2}+\frac{\gamma}{16} u_{x x}^{2} u_{x x x x}\right)\right]
\end{aligned}
$$

For wave dispersion characteristics in the nonlinear chain with both quadratic and cubic nonlinearities, we will analyze the above partial differential equation by using the multiple scales perturbation method.

\section{B. Second-Order Multiple Scales Analysis in Continuum Limit}

We analyze the wave equation using the method of multiple scales (MMS). We assume,

$$
\begin{aligned}
u(x, t) & =u_{0}\left(x, T_{0}, T_{1}, T_{2}\right)+\epsilon u_{1}\left(x, T_{0}, T_{1}, T_{2}\right)+\epsilon^{2} u_{2}\left(x, T_{0}, T_{1}, T_{2}\right)+\cdots \\
\frac{d}{d t} & =D_{0}+\epsilon D_{1}+\epsilon^{2} D_{2}+\cdots \\
\frac{d^{2}}{d t} & =D_{0}^{2}+\epsilon\left(2 D_{0} D_{1}\right)+\epsilon^{2}\left(D_{1}^{2}+2 D_{0} D_{2}\right)+\cdots
\end{aligned}
$$

where $D_{i}=\frac{\partial}{\partial T_{i}}$. Using equation (41) in equation (40) and equating like powers of $\epsilon$ yields the following set of equations

$$
\begin{array}{r}
\epsilon^{0}: D_{0}^{2} u_{0}=2 \alpha\left(\frac{u_{0_{x x}}}{2 !}+\frac{u_{0_{x x x x}}}{4 !}+\frac{u_{0_{x x x x x x}}}{6 !}+\frac{u_{0_{x x x x x x x x}}}{8 !}\right) \\
\epsilon^{1}: D_{0}^{2} u_{1}-2 \alpha\left(\frac{u_{1_{x x}}}{2 !}+\frac{u_{1_{x x x x}}}{4 !}+\frac{u_{1_{x x x x x x}}}{6 !}+\frac{u_{1_{x x x x x x x x}}}{8 !}\right) \\
=-2 D_{0} D_{1} u_{0}+2 \beta u_{0_{x}} u_{0_{x x}}+\left(\frac{\beta}{6} u_{0_{x}} u_{0_{x x x x}}+\frac{\beta}{3} u_{0_{x x}} u_{0_{x x x}}\right)+\left(\frac{\beta}{180} u_{0_{x}} u_{0_{x x x x x}}+\frac{\beta}{60} u_{0_{x x}} u_{0_{x x x x x}}+\frac{\beta}{36} u_{0_{x x x}} u_{0_{x x x x}}\right)
\end{array}
$$




$$
\begin{aligned}
\epsilon^{2}: & D_{0}^{2} u_{2}-2 \alpha\left(\frac{u_{2_{x x}}}{2 !}+\frac{u_{2_{x x x x}}}{4 !}+\frac{u_{2_{x x x x x x}}}{6 !}+\frac{u_{2_{x x x x x x x}}}{8 !}\right) \\
& =-2 D_{0} D_{1} u_{1}-\left(D_{1}^{2}+2 D_{0} D_{2}\right) u_{0} \\
& +\left(2 \beta\left(u_{1_{x}} u_{0_{x x}}+u_{0_{x}} u_{1_{x x}}\right)\right)+\left(\frac{\beta}{6}\left(u_{1_{x}} u_{0_{x x x x}}+u_{0_{x}} u_{1_{x x x x}}\right)+\frac{\beta}{3}\left(u_{1_{x x}} u_{0_{x x x}}+u_{0_{x x}} u_{1_{x x x}}\right)\right) \\
& +\left(\frac{\beta}{180}\left(u_{1_{x}} u_{0_{x x x x x}}+u_{0_{x}} u_{1_{x x x x x x}}\right)+\frac{\beta}{60}\left(u_{1_{x x}} u_{0_{x x x x x}}+u_{0_{x x}} u_{1_{x x x x x}}\right)+\frac{\beta}{36}\left(u_{1_{x x x}} u_{0_{x x x x}}+u_{0_{x x x}} u_{1_{x x x x}}\right)\right) \\
& +3 \gamma u_{0_{x}}^{2} u_{0_{x x}}+\left(\frac{\gamma}{4} u_{0_{x}}^{2} u_{0_{x x x x}}+\gamma u_{0_{x}} u_{0_{x x}} u_{0_{x x x}}+\frac{\gamma}{4} u_{0_{x x}}^{3}\right) \\
& +\left(\frac{\gamma}{120} u_{0_{x}}^{2} u_{0_{x x x x x x}}+\frac{\gamma}{20} u_{0_{x}} u_{0_{x x}} u_{0_{x x x x x}}+\frac{\gamma}{12} u_{0_{x}} u_{0_{x x x}} u_{0_{x x x x}}+\frac{\gamma}{12} u_{0_{x x}} u_{0_{x x x}}^{2}+\frac{\gamma}{16} u_{0_{x x}}^{2} u_{0_{x x x x}}\right)
\end{aligned}
$$

We seek a traveling wave solution to equation (42) in order to solve for $z_{j_{0}}$. Let

$$
\begin{aligned}
& u_{0}=y_{0}+\bar{y}_{0}=A e^{i\left(k x-\omega_{0} T_{0}\right)}+\bar{A} e^{-i\left(k x-\omega_{0} T_{0}\right)} \\
& u_{0} \underbrace{x \ldots x}_{\text {n-times }}=(i k)^{n} y_{0}+c . c .
\end{aligned}
$$

where $y_{0}=A e^{i\left(k x-\omega_{0} T_{0}\right)}$ and $u_{0} \underbrace{x \ldots x}_{\text {n-times }}$ is the $n^{t h}$ order partial derivative of $u_{0}$ with respect to $x$. Using this assumed $u_{0}$ in equation (42) the dispersion characteristics of a linear chain is found as

$$
\omega_{0}^{2}=2 \alpha\left(\frac{k^{2}}{2 !}-\frac{k^{4}}{4 !}+\frac{k^{6}}{6 !}-\frac{k^{8}}{8 !}\right)
$$

the right hand side of of which resembles a truncated form of $2 \alpha(1-\cos (k))$.

The presence of resonant terms $e^{i\left(k x-\omega_{0} T_{0}\right)}$ in equation (43) will result in unbounded solutions. For bounded solution $u_{1}$, the secular terms in equation (43) needs to be eliminated. Hence setting the coefficients of $e^{i\left(k x-\omega_{0} T_{0}\right)}$ in $\epsilon^{1}$ equation to zero. In doing so, it is noted that

$$
u_{0} \underbrace{x \ldots x}_{\text {m-times }} u_{0}^{u_{\text {n-times }}^{x \ldots x}}=\left[(-1)^{m}+(-1)^{n}\right](i k)^{m+n} y_{0} \bar{y}_{0}+(i k)^{m+n} y_{0}^{2}+(-i k)^{m+n} \bar{y}_{0}^{2}
$$

for $m, n=1,2, \cdots$. The solvability condition found by eliminating the secular terms, leads to $D_{1} A=0$. I.e. the amplitude $A$ is independent of slow time-scale $T_{1}$. Hence removing the secular terms we simplify the equation (43) as

$$
\begin{aligned}
D_{0}^{2} u_{1} & -2 \alpha\left(\frac{u_{1_{x x}}}{2 !}+\frac{u_{1_{x x x x}}}{4 !}+\frac{u_{1_{x x x x x x}}}{6 !}+\frac{u_{1_{x x x x x x x}}}{8 !}\right) \\
& =2 \beta u_{0_{x}} u_{0_{x x}}+\left(\frac{\beta}{6} u_{0_{x}} u_{0_{x x x x}}+\frac{\beta}{3} u_{0_{x x}} u_{0_{x x x}}\right)+\left(\frac{\beta}{180} u_{0_{x}} u_{0_{x x x x x x}}+\frac{\beta}{60} u_{0_{x x}} u_{0_{x x x x x}}+\frac{\beta}{36} u_{0_{x x x}} u_{0_{x x x x}}\right) \\
& =\beta\left(2(i k)^{3}+\frac{(i k)^{5}}{2}+\frac{(i k)^{7}}{20}\right) A^{2} e^{2 i\left(k x-\omega_{0} T_{0}\right)}+c . c .
\end{aligned}
$$

The particular solution to the above equation is of the form,

$$
u_{1}=A_{1} e^{2 i\left(k x-\omega_{0} T_{0}\right)}+\text { c.c. }
$$

Using the above particular solution in the $\epsilon^{1}$ equation and balancing the coefficients of $e^{2 i\left(k x-\omega_{0} T_{0}\right)}$ lead to

$$
\left[\left(-2 i \omega_{0}\right)^{2}-2 \alpha\left(\frac{(2 i k)^{2}}{2 !}+\frac{(2 i k)^{4}}{4 !}+\frac{(2 i k)^{6}}{6 !}+\frac{(2 i k)^{8}}{8 !}\right)\right] A_{1}=\beta A^{2}\left[2(i k)^{3}+\frac{(i k)^{5}}{2}+\frac{(i k)^{7}}{20}\right]
$$

Using equation (46) in equation (51) we get

$$
8 \alpha\left[\frac{3 k^{4}}{4 !}-\frac{15 k^{6}}{6 !}+\frac{63 k^{8}}{8 !}\right] A_{1}=i \beta A^{2}\left[2 k^{3}-\frac{k^{5}}{2}+\frac{k^{7}}{20}\right]
$$

and further simplifying

$$
A_{1}=\frac{2 i \beta}{\alpha k}\left[1-\frac{k^{2}}{4}+\frac{k^{4}}{40}\right]\left[1-\frac{k^{2}}{6}+\frac{k^{4}}{80}\right]^{-1} A^{2}
$$

The solution to $\epsilon^{1}$ equation is therefore

$$
u_{1}=\frac{2 i \beta}{\alpha k}\left[1-\frac{k^{2}}{4}+\frac{k^{4}}{40}\right]\left[1-\frac{k^{2}}{6}+\frac{k^{4}}{80}\right]^{-1} A^{2} e^{2 i\left(k x-\omega_{0} T_{0}\right)}+\text { c.c. }
$$


Letting $\tilde{A}_{1}=\frac{2 i \beta}{\alpha k}\left[1-\frac{k^{2}}{4}+\frac{k^{4}}{40}\right]\left[1-\frac{k^{2}}{6}+\frac{k^{4}}{80}\right]^{-1}$, we get $u_{1}=\tilde{A}_{1} y_{0}^{2}+c . c$. , and $u_{1} \underbrace{x \ldots x}_{\text {m-times }}=(2 i k)^{m} \tilde{A}_{1} y_{0}^{2}+c . c .$. Since $u_{0}$ and $u_{1}$ are independent of $T_{1},\left(-2 D_{0} D_{1} u_{1}\right)$ and $\left(D_{1}^{2} u_{0}\right)$ in equation (44) vanish. Also

$$
\begin{aligned}
& u_{1} \underbrace{x \ldots x}_{\text {m-times }} u^{u_{1} \ldots x}=\left[2^{m}(i k)^{m+n} \tilde{A}_{1} y_{0}^{3}+(2 i k)^{m}(-i k)^{n} \tilde{A}_{1} y_{0}^{2} \bar{y}_{0}\right]+\text { c.times } \\
& u_{0} \underbrace{x \ldots x}_{\text {1-times }} u_{0}^{u_{\text {m-times }}^{x \ldots x}} u_{0}^{x_{\text {n-times }}^{x \ldots x}}=\left[(i k)^{l+m+n}\left[(-1)^{l}+(-1)^{m}+(-1)^{n}\right] y_{0}^{2} \bar{y}_{0}+(i k)^{l+m+n} y_{0}^{3}\right]+\text { c.c. }
\end{aligned}
$$

where $l, m, n=1,2, \cdots$.

Using the above relations in equation (44), it is clear that the only terms that contribute to the secularity. Hence, the coefficients of $e^{i\left(k x-\omega_{0} T_{0}\right)}$, are the coefficients of $y_{0}^{2} \bar{y}_{0}$ multiplied by $A^{2} \bar{A}$. Substituting $u_{0}$ and $u_{1}$ into equation (44), the solvability condition is found as bellow:

$$
2\left(i \omega_{0}\right)\left(D_{2} A\right)=\beta\left[4(i k)^{3}+(i k)^{5}+\frac{1}{10}(i k)^{7}\right] \tilde{A}_{1} A^{2} \bar{A}+\gamma\left[3(i k)^{4}+\frac{1}{2}(i k)^{6}+\frac{3}{80}(i k)^{8}\right] A^{2} \bar{A}
$$

Letting $A=\frac{1}{2} d\left(T_{2}\right) e^{-i \hat{\theta}\left(T_{2}\right)}$, and using the relations $A^{2} \bar{A}=\frac{1}{8} d^{3} e^{-i \hat{\theta}}$ and $2 D_{2} A=\left(d^{\prime}-d i \hat{\theta}^{\prime}\right) e^{-i \hat{\theta}}$, where $d^{\prime}=\frac{\partial d}{\partial T_{2}}$, and $b^{\prime}=\frac{\partial \hat{\theta}}{\partial T_{2}}$, the above equation becomes

$$
\left(i \omega_{0} d^{\prime}+\omega_{0} d \hat{\theta}^{\prime}\right) e^{-i \hat{\theta}}=\frac{3 \gamma k^{4}}{8}\left[1-\frac{k^{2}}{6}+\frac{k^{4}}{80}\right] d^{3} e^{-i \hat{\theta}}+\frac{\beta^{2} k^{2}}{\alpha}\left[1-\frac{k^{2}}{4}+\frac{k^{4}}{40}\right]^{2}\left[1-\frac{k^{2}}{6}+\frac{k^{4}}{80}\right]^{-1} d^{3} e^{-i \hat{\theta}}
$$

Equating the real and imaginary parts, expanding the square, and using the Maclaurin series expansion $(1-x)^{-1}=\sum_{n=0}^{\infty} x^{n}$, the solvability condition is found as

$$
\begin{array}{ll}
\text { Im: } \quad d^{\prime}=0 \\
\text { Re: } \quad \hat{\theta}^{\prime}=\left[\frac{3 \gamma k^{4}}{8}\left[1-\frac{k^{2}}{6}+\frac{k^{4}}{80}\right]+\frac{\beta^{2} k^{2}}{\alpha}\left[1-\frac{k^{2}}{3}+\frac{2 k^{4}}{45}\right]\right] \frac{d^{2}}{\omega_{0}}
\end{array}
$$

Solving the equation (57), making the integration constant zero without loss of generality, and noting that $T_{2}=\epsilon^{2} T_{0}$, the slowly varying amplitude $(d)$ and phase $(\hat{\theta})$ becomes,

$$
\begin{aligned}
& d=d_{0} \\
& \hat{\theta}=\left[\frac{3 \gamma k^{4}}{8}\left[1-\frac{k^{2}}{6}+\frac{k^{4}}{80}\right]+\frac{\beta^{2} k^{2}}{\alpha}\left[1-\frac{k^{2}}{3}+\frac{2 k^{4}}{45}\right]\right] \frac{a_{0}^{2}}{\omega_{0}} \epsilon^{2} T_{0}
\end{aligned}
$$

Therefore combining equation (45) with $A=\frac{1}{2} d e^{-i \hat{\theta}}$, the frequency $\omega$ can be written as

$$
\omega=\omega_{0}+\frac{\hat{\theta}}{T_{0}}=\omega_{0}\left[1+\frac{\epsilon^{2} d_{0}^{2}}{\omega_{0}^{2}}\left\{\frac{3 \gamma k^{4}}{8}\left[1-\frac{k^{2}}{6}+\frac{k^{4}}{80}\right]+\frac{\beta^{2} k^{2}}{\alpha}\left[1-\frac{k^{2}}{3}+\frac{2 k^{4}}{45}\right]\right\}\right]
$$

Also note that, in the above dispersion relationship, $\omega_{0}$ is a function of the wave number $k$. We find that applying the Taylor series expansion of $\sin ^{2} k$ and $\sin ^{2} \frac{k}{2}$ and taking the first three terms in the expansion will result in the equation (59). Hence, in the limit, the frequency in the displacement coordinate system can be accurately represented as

$$
\omega\left(k, d_{0}\right)=\omega_{0}\left[1+\frac{\epsilon^{2} d_{0}^{2}}{\omega_{0}^{2}}\left\{\frac{3 \gamma}{8}(2(1-\cos k))^{2}+\frac{\beta^{2}}{\alpha} \sin ^{2} k\right\}\right]
$$

Further simplifying above equation, the dispersion relation in the limiting condition is

$$
\omega\left(k, d_{0}\right)=\omega_{0}\left[1+\frac{3 \epsilon^{2} d_{0}^{2}}{4 \alpha}\left\{\gamma(1-\cos k)+\frac{2 \beta^{2}}{3 \alpha}(1+\cos k)\right\}\right]
$$

Hence our quest to solve for the dispersion characteristics in a continuum with quadratic and cubic nonlinearities using method of multiple scales in a partial differential equation has led us to the dispersion relations as shown in equation (61), which is an exact match with the dispersion relation obtained in equation (32) of Section II by applying multiple scales to the discrete chain of mass spring system.

\section{Results AND Discussion}

Assuming $x_{j \pm 1}=x_{j} \pm h$, and letting $x_{0}=0$ without loss of generality, we have $x_{j}=j h$. The non-dimensional wave number is then $\mu=k h$, where $k$ is the wave number and $h$ is the spacial distance between the adjacent masses. Hence, 


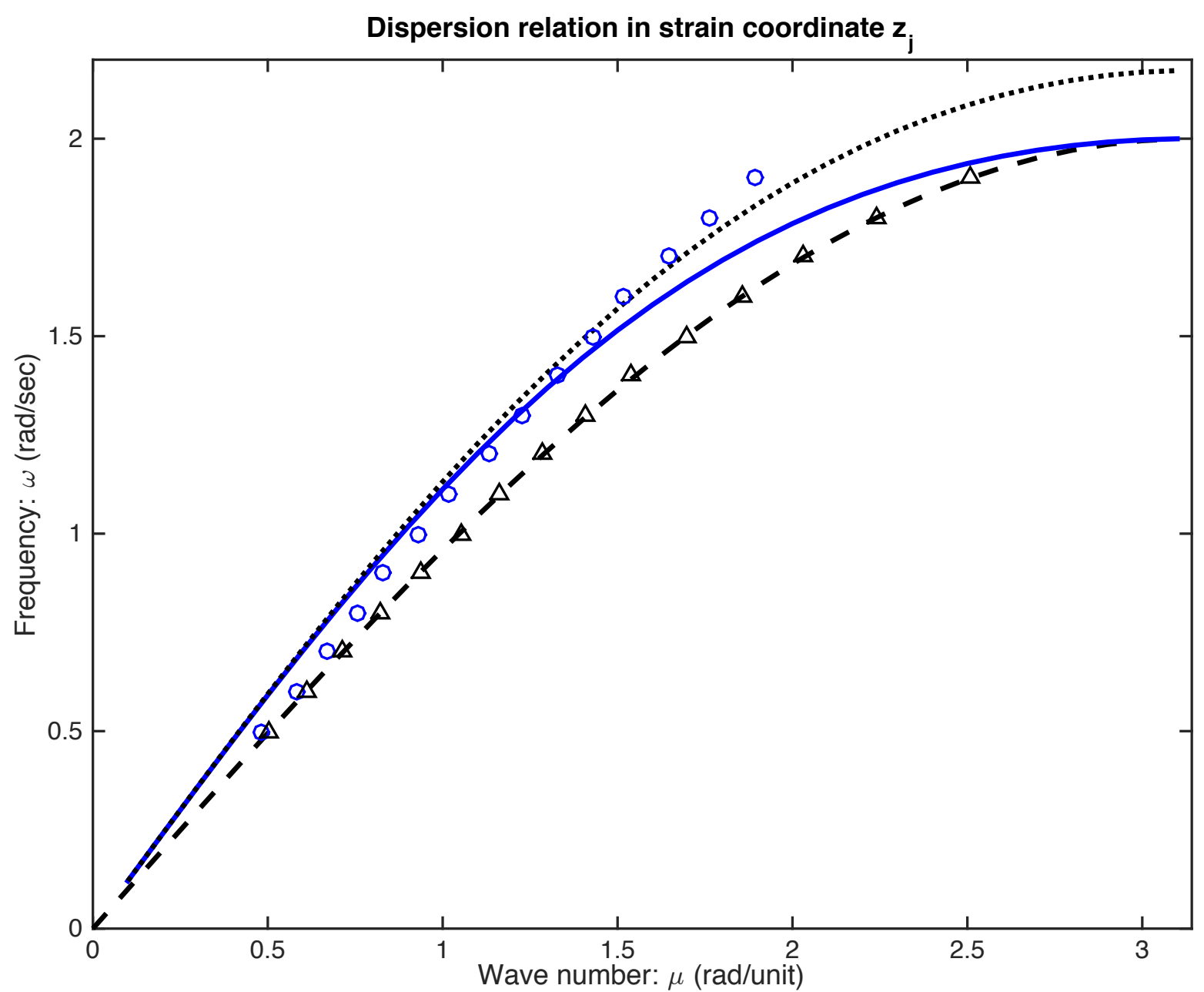

Fig. 3: The wave dispersion characteristics in linear and nonlinear chains with weak quadratic nonlinearity $(\epsilon=0.3)$ when a sinusoidal wave of amplitude $d_{0}=0.8$ is propagated down a chain connected by weakly nonlinear spring elements. Here the horizontal axis represents the wave number $(\mu)$ and the vertical axis represents the frequency $\omega$ which is a function of both the wave number $\mu$ and the amplitude of oscillation $d_{0}$. The solid (blue) curve corresponds to the quadratic nonlinear case where $\alpha=1, \beta=1.9$, and $\gamma=0$. The dotted (black) curve corresponds to the quadratic plus cubic case where $\alpha=1, \beta=1.9$, and $\gamma=1$. The dashed (black) curve corresponds to the linear case where $\alpha=1, \beta=0$, and $\gamma=0$. The circles represent the numerical results for the case where $\alpha=1, \beta=1.9$, and $\gamma=0$, and the triangles depict numerical results for the linear case.

substituting $\mu=k h$ into equation (27) and equation (13), the nondimesional form of the dispersion equation becomes

$$
\omega=\sqrt{2 \alpha(1-\cos \mu)}\left[1+\frac{3 \epsilon^{2} a_{0}^{2}}{8 \alpha}\left(\gamma+\frac{2 \beta^{2}(1+\cos \mu)}{3 \alpha(1-\cos \mu)}\right)\right]
$$

Equations (32) and equivalently (61) relate the wave frequency to the wave number, and therefore provide the fundamental relationship describing the dispersion properties. Descriptions of phase velocity and group velocity can be derived from this equation. An illustration of the quadratic effect on the dispersion relation is shown in Figure 3 . The solid curve shows the case of $\gamma=0$, isolating the effect of quadratic nonlinearity, with $\beta=1.9, \epsilon=0.3$, and $d_{0} \approx 0.8$. The dotted curve includes the addition of hardening cubic nonlinearity, with $\gamma=1$, while the dashed line shows the linear dispersion relation for reference. The figure shows that the quadratic nonlinearity tends to increase the wavelength of a wave of a given frequency (equivalently, increase the frequency of waves of a given wave number). This effect occurs with both positive and negative quadratic coefficients, as the contribution goes with $\beta^{2}$ in equation (32). The theory then suggests that addition of hardening cubic nonlinearity tends to further increase the frequency of waves of a given wavelength, while softening cubic nonlinearity would decrease the frequency.

Simulation approximations have been made in order to see if the trends predicted by the second-order perturbation analysis have merit. The analysis is for a freely propagating harmonic wave approximation. There are challenges with these simulations. With nonlinearity, it is difficult generate a freely propagating pure waveform. As such, we have simulated an imposed harmonic motion in the chain (at the zeroth mass), and observed the propagation to the right, with the assumption that the propagating wave 


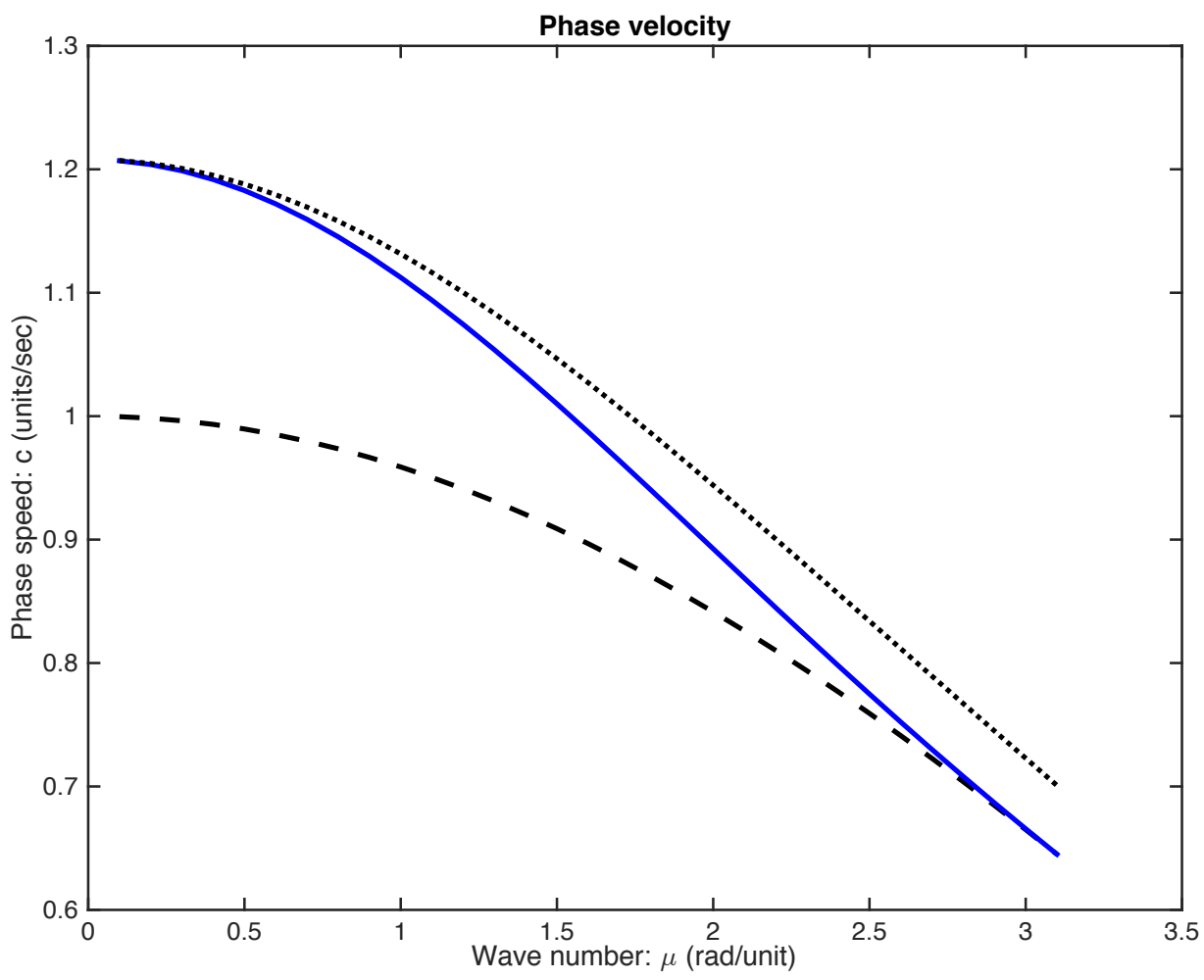

Fig. 4: The wave speed or the phase velocity, $c$, shown with respect to the wave number, $\mu$, at an amplitude $d_{0}=0.8$ and $\epsilon=0.3$. The solid curve corresponds to the linear and the dashed curve correspond to quadratic nonlinear case where $\alpha=1$, $\beta=1.9$, and $\gamma=0$. The dotted data points represent the numerical results computed by taking the ratio of the numerical frequency and numerical wave number.

will settle into a waveform that nearly follows the free wave. A similar approach was used in reference [10] for observations of cubic effects. In our simulation, we used $N=100$ masses, and monitored the wave response through approximately the first $N / 2$ masses next to the imposed zeroth mass, but prior to reflections returning to the measurement zone. Given a wave frequency, we estimated the wavenumber of a snapshot of the response in this finite measurement zone in the spatial domain over several wavelengths, the number of which was determined by how the spatial sampling allowed the periodicity of the waves to match up.

We simulated the system in the $z_{j}$ coordinates (relative deflections, or strain), since the responses are better suited for signal analysis. We aimed to simulate with a uniform displacement amplitude. The associated strain amplitude thus depends on the wave number (equivalently, the wave speed). We approximated the appropriate strain amplitude based on the first of equations 31. As the frequency (and hence wave number) increases under a fixed displacement amplitude, the strain amplitude increases, which risks pushing the small $\epsilon$ asymptotics. The perturbation analysis is done on strain variables, $z_{j}$, and is valid for small strains. As the frequency decreases, we observed that the waveform takes on more complicated harmonics in its content, and it became difficult to estimate wave numbers in our finite-range simulation. The simulation observations are plotted as circles and compared to the predicted dispersion curve in Figure 3 for the case of $\gamma=0$, isolating the effect of quadratic nonlinearity, with $\beta=1.9, \epsilon=0.3$, and $d_{0} \approx 0.8$. The predicted trends of the dispersion relation are well represented in the middle range of wave frequencies. The case of $\epsilon=0.2$ (plot not included) shows a smaller quadratic effect, but the simulations match with the prediction for a larger middle range of frequencies than in the case of $\epsilon=0.3$. Although the asymptotic analysis is for small $\epsilon$, the simulations show that as we push the value of $\epsilon$, the behavior persists, and becomes more enhanced, although the range of prediction for single-harmonic waves is reduced.

For reference, simulations of the linear system excited in the same manner produced nearly harmonic waves in the vicinity of the zeroth mass, with characteristics of frequency versus wave number that match the linear dispersion curve (see the triangle symbols in Figure 3).

The phase velocity or the wave speed $c$ and the group velocity $v_{g}$ are functions of the wave number $\mu$ and the wave amplitude $d_{0}$ and can be found from the following relation between the frequency and wave number based on equation (62): 


$$
\begin{aligned}
c\left(\mu, d_{0}\right) & =\frac{\omega\left(\mu, d_{0}\right)}{\mu} \\
v_{g}\left(\mu, d_{0}\right) & =\frac{d \omega\left(\mu, d_{0}\right)}{d \mu}=\frac{\alpha \sin \mu}{\omega_{0}}\left[1+\frac{3 \epsilon^{2} d_{0}^{2}}{4 \alpha}\left\{\gamma(1-\cos \mu)+\frac{2 \beta^{2}}{3 \alpha}(1+\cos \mu)\right\}\right]+\omega_{0}\left[\frac{3 \epsilon^{2} d_{0}^{2}}{4 \alpha} \sin \mu\left\{\gamma-\frac{2 \beta^{2}}{3 \alpha}\right\}\right]
\end{aligned}
$$

where $\omega_{0}=\sqrt{2 \alpha(1-\cos \mu)}$.

At fixed amplitude, the phase velocity is higher for the quadratic nonlinear case at smaller wave numbers as compared to the linear chain as seen in Figure 4. At fixed amplitude $d_{0}=0.8$, the group velocity, shown in Figure 5, is found to be higher at lower spatial frequencies and lower at higher spatial frequencies in the quadratic nonlinear case as compared to the linear case.

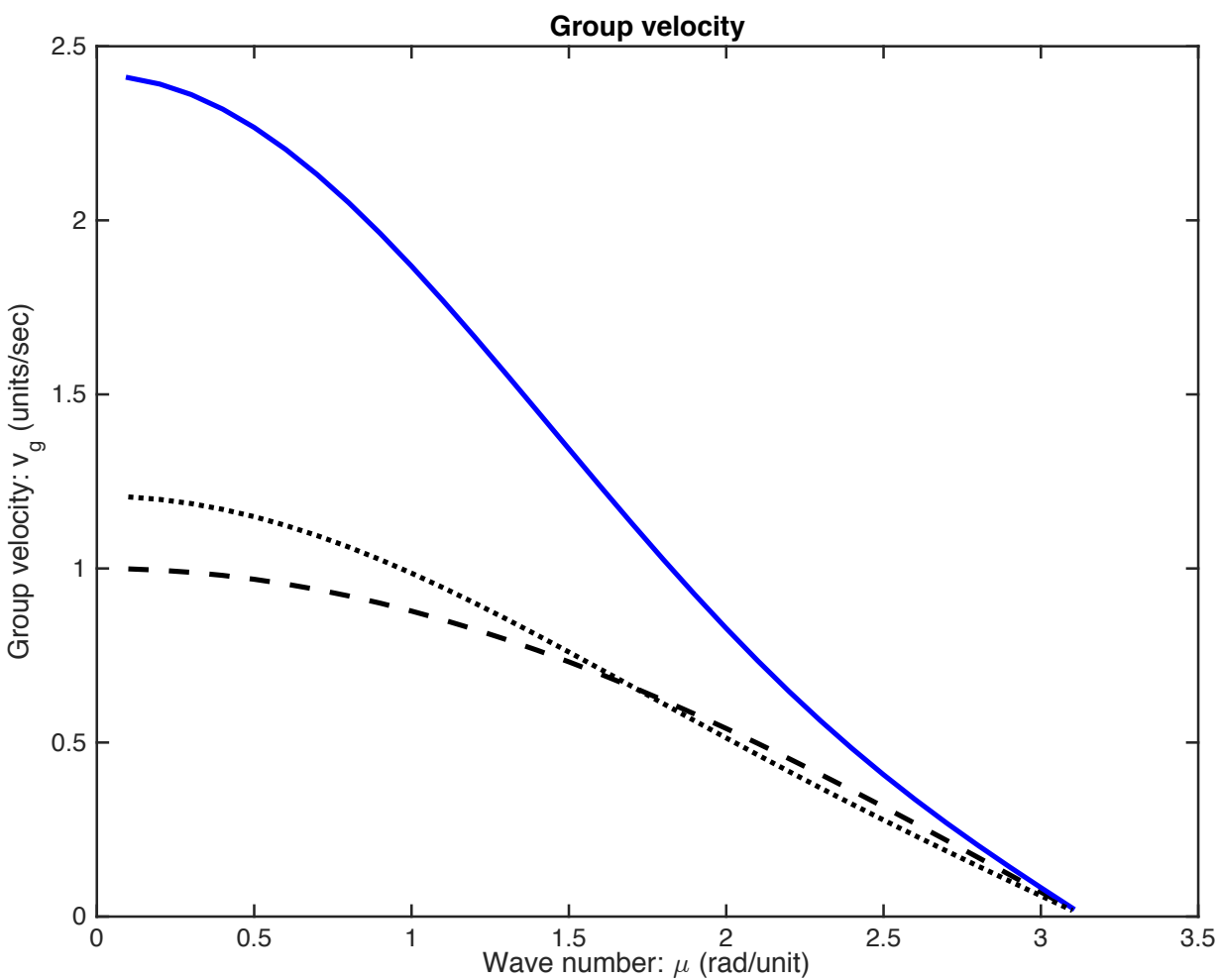

Fig. 5: The non-dimensional wave number, $\mu$, dependent group velocity, $v_{g}$, at an amplitude $d_{0}=0.8$ and $\epsilon=0.2$ shows the wave velocities in the linear and nonlinear chains. The solid curve corresponds to the linear case and the dashed curve correspond to quadratic nonlinear case where $\alpha=1, \beta=1.9$, and $\gamma=0$. The dotted data points represent the numerical results.

\section{Vi. Propagation And Attenuation}

Attenuation can occur in space or time. Attenuation in general is a reduction of signal strength during transmission. In the case of temporal attenuation, the frequency is complex, interpreted as damping, and energy is lost as time evolves. In the case of spatial attenuation, the wave number $\mu$ is a complex number such that $\mu=\rho+i \sigma$. This geometric attenuation is not associated with energy loss, although transmission capability is reduced.

Squaring the frequency and wave-number characteristics in equation (62) and neglecting the order of $\epsilon^{4}$, we solve for the wave number $\mu$, as a function of the frequency $\omega$ (using Mathematica), resulting in a complex wave number $\mu$. The real and imaginary parts of this complex wave number are the propagation and the attenuation constants respectively. The expressions are complicated and piecewise defined and are not presented here, but are plotted in Figure 6.

A traveling wave is represented by

$$
u=A e^{i[\mu j-\omega t]}
$$

For complex wave number $\mu=\rho+i \sigma$, where $\rho$ is the propagation constant and $\sigma$ is the attenuation constant, we have

$$
u=A e^{i[(\rho+i \sigma) j-\omega t]}=A e^{-\sigma x} e^{i[\rho j-\omega t]}
$$


So there is a traveling wave for non-zero $\rho$ but with a reducing amplitude, $A e^{-\sigma j}$, in space. A smaller $\sigma$ means a lower attenuation of signal strength. When $\sigma=0$, we have a traveling wave without spatial reduction in amplitude, i.e. no attenuation.

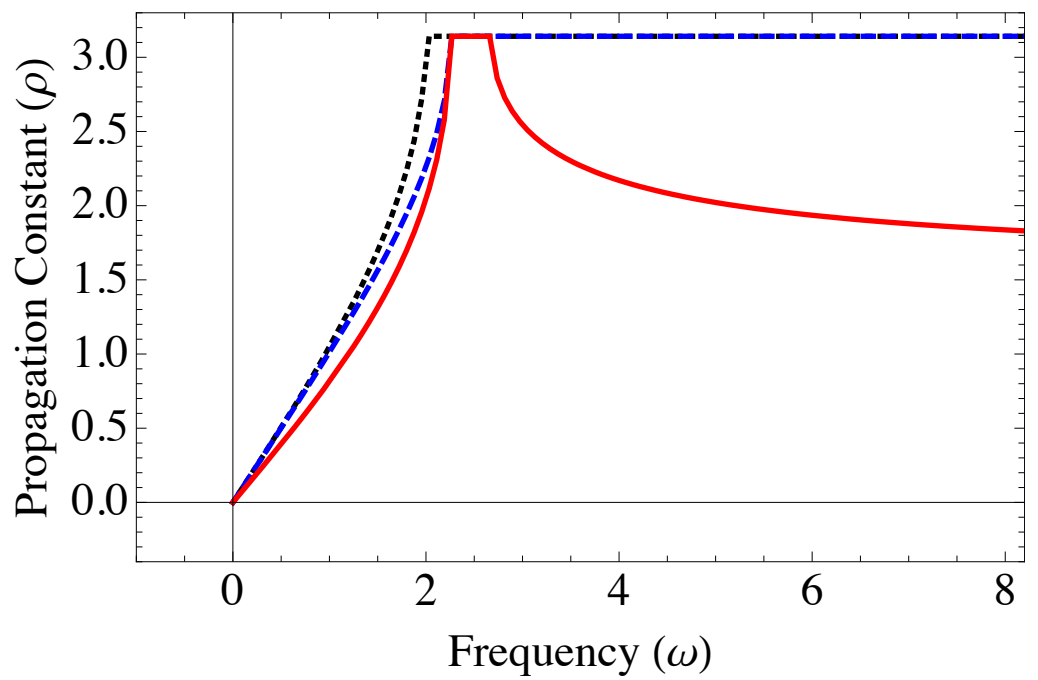

(a)

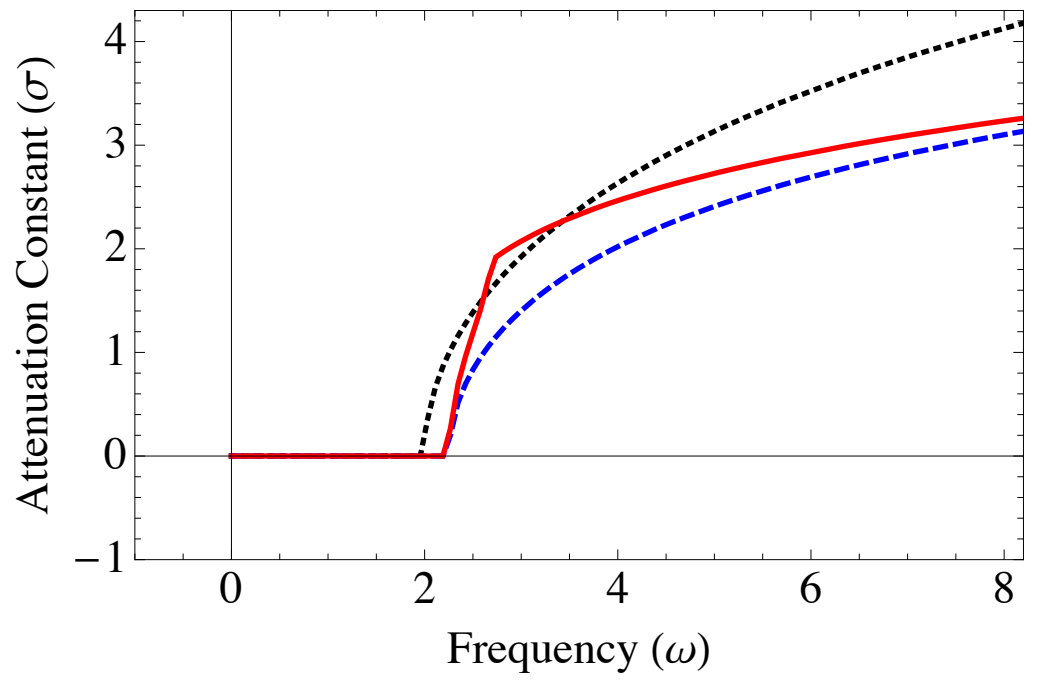

(b)

Fig. 6: (a) The propagation constant, $\rho$, and (b) the attenuation constant, $\sigma$, for a particular amplitude $d_{0}=1$ and $\epsilon=0.3$. The dotted, dashed and solid curves correspond to the linear $(\alpha=1, \beta=0, \gamma=0)$, cubic $(\alpha=1, \beta=0, \gamma=1)$, and quadratic $(\alpha=1, \beta=1.9, \gamma=1)$ chains respectively.

From Figure 6 we observe that the cubic, and the quadratic cases have the same cut-off frequencies ( $\omega$ ) for propagation (non-attenuated wave) without attenuation, i.e. when frequency $\omega$ lies between 0 and 2.2539 , which is consistent with dispersion plot in Figure 3. For frequencies $\omega=0$ to $\omega=2.2539$, the waves in the chain propagate with varying phases but with zero attenuation. I.e. the waves propagate without losing any energy or strength.

The propagation constant $\rho$, for the linear and the nonlinear cases with both quadratic and cubic nonlinearity, have a saturation value of $\rho=\pi$ as seen in Figure 6 (a). However, in the quadratic chain the propagation constant starts decreasing for frequencies higher than $\omega \approx 2.6545$ and eventually converges to $\rho=1.571$ for high frequencies. This means that the wave propagates with varying phases for the quadratic case for frequencies greater than $\omega=2.6545$, with sharp change in phases $\rho$ close to $\omega=2.6545$. In the quadratic case there exists a band-of frequencies from $\omega=2.2539$ to $\omega=2.6545$ where the waves propagate with constant phase while attenuating continuously until the frequency reaches the value of $\omega=2.6545$.

Also the attenuation constant, $\sigma$, in the quadratic chain is higher than the cubic chain in the frequency band $\omega=2.2539$ to $\omega=2.6545$ as seen in the Figure 6 (b). After the kink the increase in attenuation with $\omega$ is reduced. In the frequency range of $\omega=0$ and $\omega=2.2539$ there is no attenuation, and the dispersion follows that seen Figure 3 , in this case for the mixed quadratic and cubic example. 
Figure 7 shows the change in amplitude of the propagating wave when the frequency is increased for a particular wave number $\mu=0.7$. As the amplitude increase only frequencies, $\omega$, larger than 0.7 can propagate through the chain and other lower frequencies frequencies are blocked. Similarly, for frequency $\omega=1.3$, the wave numbers generated by the injected wave are limited to $\mu \approx 1.4$ for varying amplitude of the injected wave as in figure 8 . Also, the wave number exponentially approaches zero when the wave amplitude is increased indicating large wave lengths generated for larger amplitude.

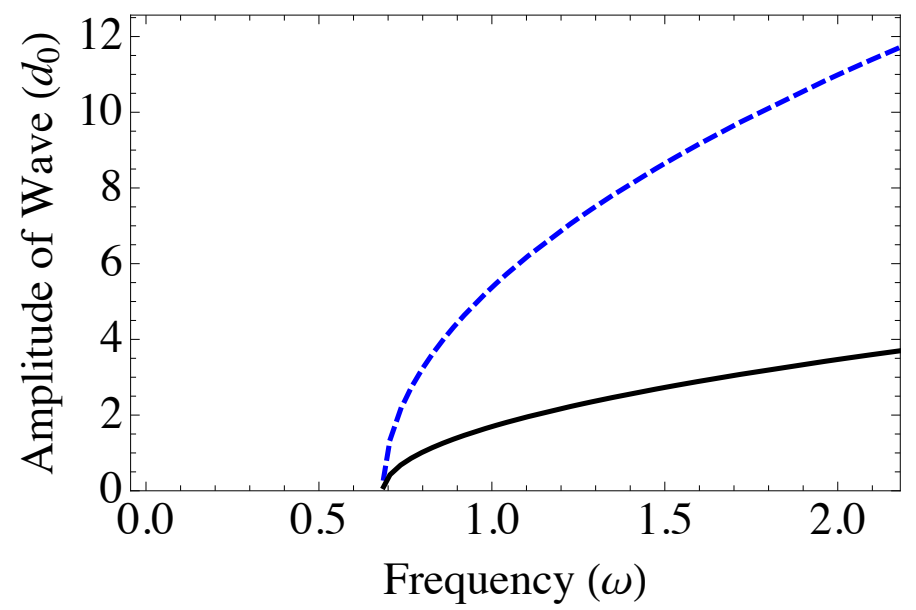

Fig. 7: The amplitude relation of the waves to the frequency $(\omega)$ for a particular wave number $\mu=0.7$. The dashed and solid curves correspond to the cubic $(\alpha=1, \beta=0, \gamma=1)$, and quadratic $(\alpha=1, \beta=1.9, \gamma=1)$ chains respectively.

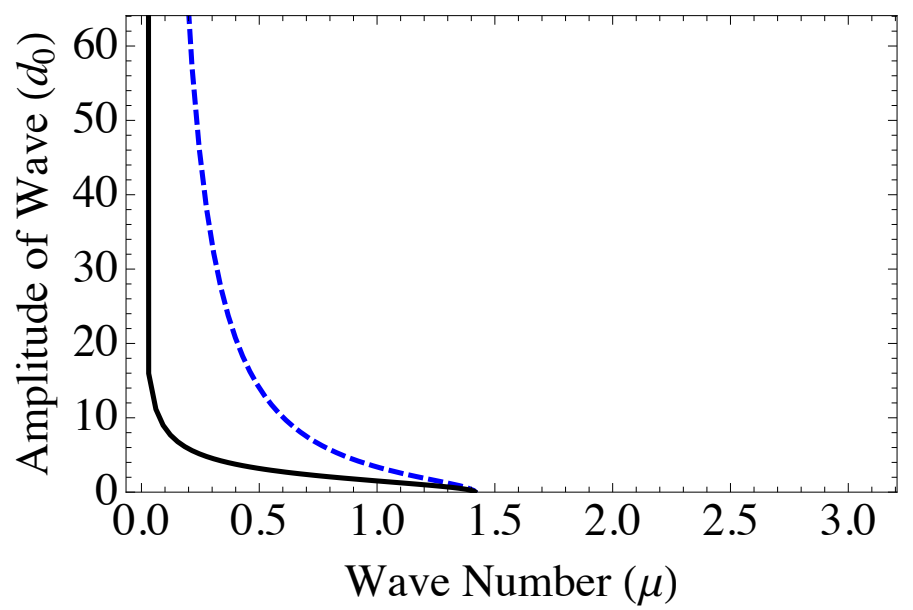

Fig. 8: The amplitude relation of the waves to the wave number $(\mu)$ for a particular frequency $\omega=1.3$. The dashed and solid curves correspond to the cubic $(\alpha=1, \beta=0, \gamma=1)$, and quadratic $(\alpha=1, \beta=1.9, \gamma=1)$ chains respectively.

\section{CONCLUSIONS}

In this paper we have presented a detailed study of traveling wave behavior in an infinite periodic chain of nonlinear elements with low-amplitude oscillations. We adapted a second-order multiple-scales analysis to accommodate traveling waves in a chain with both quadratic and cubic nonlinearities. This analysis uncovered dispersion relationships, and the effects of quadratic and cubic nonlinearities in a chain. A continuum approach has also been used to derive and confirm the validity of the analytical approximation. The quadratic effect was then demonstrated through numerical simulations in the strain coordinates.

A comparison between the quadratic (with nonzero linear and zero cubic terms) and linear cases shows that the quadratic terms lead to higher frequencies given the wavenumber. At lower wave numbers, the quadratic case has higher phase and group velocities than the linear case. At higher wave numbers, however, the group velocities for the quadratic case are lower as compared to the linear case. The effect of quadratic nonlinearity on spatial attenuation was also examined in the analysis.

\section{ACKNOWLEDGMENTS}

This material is based upon work supported by the National Science Foundation under Grant No. CMMI-1030377. Any opinions, findings, and conclusions or recommendations expressed in this material are those of the authors and do not necessarily reflect the views of the National Science Foundation. 


\section{REFERENCES}

[1] Page, J., Sukhovich, A., Yang, S., Cowan, M., van der Bi-est, F., Tourin, A., Fink, M., Liu, Z., Chan, C., and Sheng, P., 2004. "Phononic crystals". Solid State Physics, 241(15), pp. 3454-3462.

[2] Bertoldi, K., and Boyce, M., 2008. "Mechanically triggered transformations of phononic band gap elastomeric structures". Physical Review B, 77(052105).

[3] Jang, J. H., Ullal, C. K. U., Gorishnyy, T., Tsukruk, V. V., and Thomas, E. L., 2006. "Mechanically tunable three-dimensional elastomeric network/air structures via interference lithography". Nano Letters, 6(4), pp. 740-743.

[4] Liang, B., Yuan, B., and Cheng, J. C., 2009. "Acoustic diode: Rectification of acoustic energy flux in one-dimensional systems". Physical Review Letters, 13, p. 104301.

[5] Olsson, R. H. I., El-Kady, I. F., Su, M. F., Tuck, M. R., and Fleming, J. G., 2008. "Microfabricated vhf acoustic crystals and waveguides". Sensors and Actuators A: Physical, 20(1), pp. 87-93.

[6] Olsson, R. H. I., and El-Kady, I., 2009. "Microfabricated phononic crystal devices and applications". Measurement Science and Technology, 20(1), p. 012002.

[7] Sigmund, O., and Jensen, J. S., 2003. "Systematic design of phononic band-gap materials and structures by topology optimization". Philosphical Transactions of the Royal Society A: Mathematical, Physical and Engineering Sciences, 361(1806), pp. 1001-1019.

[8] Yun, Y., Miao, G. Q., Zhang, P., Huang, K., and Wei, R. J., 2005. "Nonlinear acoustic wave propagating in one-dimensional layered system". Physics Letters A, 343(5), pp. 351-358.

[9] Marathe, A., and Chatterjee, A., 2005. "Wave attenuation in nonlinear periodic structures using harmonic balance and multiple scales.". Journal of Sound and Vibration, 289(4-5), pp. 871-888.

[10] Narisetti, R. K., Leamy, M. J., and Ruzzene, M., 2010. "A perturbation approach for predicting wave propagation in one-dimensional nonlinear periodic structures". ASME Journal of Vibration and Acoustics, 132(3), p. 031001.

[11] Narisetti, R. K., Ruzzene, M., and Leamy, M. J., 2011. "A perturbation approach for analyzing dispersion and group velocities in two-dimensional nonlinear periodic lattices". ASME Journal of Vibration and Acoustic, 133(6), p. 061020.

[12] Nayfeh, A. H., and Rice, M. H., 1972. "On the propagation of disturbances in a semi-infinite one-dimensional lattice". American Journal of Physics, 40(3), pp. 469-470.

[13] Daraio, C., Nesterenko, V. F., Herbold, E. B., and Jin, S., 2005. "Strongly nonlinear waves in a chain of teflon beads". Physical Review E, 72(1), p. 016603

[14] Peyrard, M., and Kruskal, M. D., 1984. "Kink dynamics in the highly discrete sine-gordon system". Physica D, 14(1), pp. 88-102.

[15] Vakakis, A. F., and King, M. E., 1995. "Nonlinear wave transmission in a monocoupled elastic periodic system". Journal of Acoustical Society of America, 98(3), pp. 1534-1546.

[16] Vakakis, A. F., and King, M. E., 1998. "Resonant oscillations of a weakly coupled, nonlinear layered system". Acta Mechanica, 128(1), pp. 59-80.

[17] Asfar, O. R., and Nayfeh, A. H., 1983. "The application of the method of multiple scales to wave propagation in periodic structures". Society of Industrial and Mathematics Review, 25(4), pp. 455-480.

[18] Chakraborty, G., and Mallik, A. K., 2001. "Dynamics of a weakly nonlinear periodic chain". International Journal of Non-linear Mechanics, 36(2), pp. 375-389.

[19] Rushchitsky, J. J., and Saveleva, E. V., 2006. "On the interaction of cubically nonlinear transverse plane waves in an elastic material". International Applied Mechanics, 42(6), pp. 661-668.

[20] Rothos, V. M., and Vakakis, A. F., 2009. "Dynamic interactions of traveling waves propagating in a linear chain with an local essentially nonlinear attachment". Wave Motion, 46(3), pp. 174-188.

[21] I., H. M., Hulbert, G., and Scott, R., 2002. "Tailoring of wave propagation characteristics in periodic structures with multilayer unit cells". Proceedings of 17 th American Society of Composites Technical Conference.

[22] I., H. M., Hamza, K., Hulbert, G., and Saitou, K., 2007. "Optimal synthesis of 2d phononic crystals for broadband frequency isolation". Waves Random Complex Media, 17(4).

[23] Diaz, A., Haddow, A., and Ma, L., 2005. "Design of band-gap grid structures”. Structural and Multidisciplinary Optimization, 29(6), pp. 418-431.

[24] Manktelow, K., Leamy, M. J., and Ruzzene, M., 2011. "Multiple scales analysis of wave-wave interactions in a cubically nonlinear monoatomic chain". Nonlinear Dynamics, 63, pp. 193-203.

[25] Panigrahi, S. R., Feeny, B. F., and Diaz, A. R., 2014. "Degenerate "star" bifurcations in a twinkling oscillator". Journal of Vibration and Acoustics, 136(2), p. 021004.

[26] Panigrahi, S. R., Feeny, B. F., and Diaz, A. R., 2014. “"Eclipse' bifurcation in a twinkling oscillator”. Journal of Vibration and Acoustics, 136(3), p. 034504 